\title{
Indução de estro em cabras da raça Toggenburg com dois diferentes dispositivos intravaginais
}

[Estrous induction in Toggenburg goats using intravaginal devices]

\author{
V.V. Maffili ${ }^{1}$, C.A.A. Torres ${ }^{2}$, J.H. Bruschi ${ }^{3}$, J.F. Fonseca ${ }^{4}$, J.H.M. Viana ${ }^{3}$ \\ ${ }^{1}$ Centro de Pesquisa Gonçalo Muniz - Fiocruz \\ Rua Waldemar Falcão, 121 - Brotas \\ 40295-001 - Salvador, BA \\ ${ }^{2}$ Departamento de Zootecnia - UFV, MG \\ ${ }^{3}$ Embrapa-Gado de Leite - Juiz de Fora, MG \\ ${ }^{4}$ Embrapa-Caprinos - Sobral, CE
}

\begin{abstract}
RESUMO
Doze cabras nulíparas da raça Toggenburg foram distribuídas em dois tratamentos (T1 e T2) para indução do estro. O T1 $(\mathrm{n}=6)$ consistiu na inserção da esponja impregnada com $60 \mathrm{mg}$ de acetato de

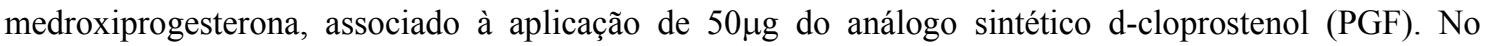
quarto dia, foram aplicadas 250UI de gonadotropina coriônica eqüina (eCG) e, no quinto, a esponja foi retirada. O T2 $(n=6)$ diferiu somente quanto ao dispositivo empregado, que foi o CIDR-G ${ }^{\circledR}$. Seis cabras do T1 e seis do T2 entraram em estro depois da retirada dos dispositivos, sendo que $83,3(5 / 6)$ e $33,3 \%$ (2/6) ficaram gestantes em T1 e T2, respectivamente. O intervalo da retirada do dispositivo ao início do estro não diferiu entre tratamentos $(\mathrm{P}>0,05)$. As características ovulatórias não foram influenciadas pelos tratamentos. $\mathrm{O}$ intervalo da retirada do dispositivo à ovulação, para ambos os protocolos de indução do estro, não diferiu $(\mathrm{P}>0,05)$ entre tratamentos. Ambos os dispositivos foram eficientes em induzir o estro.
\end{abstract}

Palavras-chave: caprino, CIDR-G, eCG, esponja

\begin{abstract}
Twelve Toggenburg breed nuliparous female goats were randomly assigned to two treatments intended to induce estrus. On day zero, animals on $T 1(n=6)$ were treated with an intravaginal sponge impregnated with 60mg of medroxyprogesterone acetate plus 50 $\mathrm{gg}$ of PGF analogue. The T1 goats received eCG (250IU) on day 4, and on day 5 the sponge was removed. The T2 goats $(n=6)$ received $C I D R-G^{\circledR}$ as

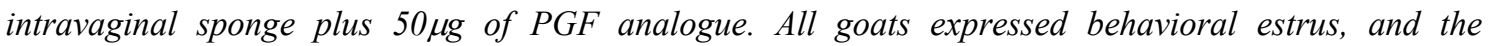
pregnancy rates were 83.3 (5/6) and 33.3\% (2/6) for $T 1$ and T2, respectively. The interval from withdrawal of the device to onset of estrus did not differ between treatments $(P>0.05)$. Ovulatory traits also were not affected by treatments. The interval between $C I D R-G^{\circledR}$ removal and ovulation for T1 and $T 2$ goats were similar $(P>0.05)$. Both sponge and CIDR-G were equally efficient in inducing the estrus behavior in goats.
\end{abstract}

Keywords: caprine, $C I D R-G$, eCG, sponge

Recebido em 21 de outubro de 2004 Aceito em 14 de setembro de 2005 E-mail: vmaffili@cpqgm.fiocruz.br Apoio: CAPES e FAPEMIG 


\section{INTRODUÇÃO}

$\mathrm{Na}$ Região Sudeste do Brasil, as cabras apresentam estacionalidade reprodutiva no período se agosto a janeiro, o que resulta em produção de leite não uniforme ao longo do ano. Com vistas a obter regularidade na produção durante todo o ano, é necessário que se faça à indução do estro. De forma geral, a indução do estro pode ser obtida pelo do uso de fotoperíodo artificial (Cordeiro, 1992), efeito do macho (Carnevali et al., 1997), uso de melatonina (Deveson et al., 1992) ou, ainda, por uma combinação de hormônios (Fonseca, 2002).

No Sudeste do Brasil, o método mais comumente utilizado para indução do estro em cabras é o fotoperíodo artificial, mas também pode ser induzido durante o anestro por protocolos que combinem a progesterona sintética ou natural, seguido por injeção intramuscular de gonadotrofina coriônica de eqüina (eCG), no momento da remoção do dispositivo. Com este propósito, várias vias são eficientes, incluindo esponjas impregnadas por progestágenos (Fonseca, 2002) CIDR-G ${ }^{\circledR}$ (Oliveira et al., 2001) e implantes para uso por via subcutânea (Oliveira et al., 2001). Basicamente, a progesterona exógena aplicada durante o período de anestro prepara o útero para a gestação e sensibiliza o eixo hipotálamo-hipófise-ovário para direta ou indiretamente responderem aos estímulos gonadotrotróficos (Foote, 1982), além de agir desbloqueando a liberação de gonadotrofinas.

Tanto a esponja intravaginal quanto o CIDR-G ${ }^{\circledR}$ têm sido utilizados com sucesso na indução do estro. Em protocolos tradicionais, os progestágenos são aplicados por longos períodos (>11 dias). No entanto, a permanência do dispositivo por períodos longos tem sido associada à baixa fertilidade (Hawk e Conley, 1972), por promover mudanças no transporte espermático, além da ocorrência de ovulação de ovócitos com qualidade inferior (Mihm et al., 1994).

Uma vez que ondas de crescimento folicular em caprinos ocorrem a cada cinco e a cada sete dias (Rubianes e Menchaca, 2003), a utilização de dispositivos liberadores de progesterona por longos períodos para indução de estro tornou-se injustificável. No entanto, não há relatos na literatura consultada, comparando os dois principais dispositivos liberadores de progesterona empregados em caprinos para indução do estro por curto período (cinco dias). Assim, o objetivo desta pesquisa foi comparar a indução de estro em cabras da raça Toggenburg com os dispositivos CIDR-G ${ }^{\circledR}$ e esponjas impregnadas com 60mg de acetato de medroxiprogesterona (MAP).

\section{MATERIAL E MÉTODOS}

O estudo foi realizado numa região localizada a $21^{\circ} 35^{\prime} \mathrm{S}, 43^{\circ} 15^{\prime} \mathrm{W}$ e a 435 metros de altitude, de clima do tipo Cwa, segundo classificação de Koppen, durante a estação de reprodução dos caprinos, no mês de maio, equivalente ao outono no hemisfério sul.

Utilizaram-se 12 cabras da raça Toggenburg, com idade média de oito meses, peso e condição de escore corporal de $(1=$ muito magra e $5=$ muito gorda). Os animais foram mantidos em confinamento total em baias coletivas, onde receberam capim napier (Panicum maximum) picado, concentrado comercial ${ }^{1}$, sal mineral $\mathrm{e}$ água ad libitum.

Os animais foram distribuídos aleatoriamente, de acordo com o peso e a condição de escore corporal, em dois tratamentos: $\mathrm{T} 1(\mathrm{n}=6)$, com indução do estro por meio da aplicação de esponjas intravaginais ${ }^{2}$ impregnadas com $60 \mathrm{mg}$ de MAP, associado a $50 \mu \mathrm{g}$ do análogo sintético d-cloprostenol $\left(\mathrm{PGF}^{3}\right)$ no dia zero e 250UI de gonadotrofina coriônica eqüina $\left(\mathrm{eCG}^{4}\right)$ no quarto dia, ambas aplicadas via intramuscular, e T2 $(n=6)$, com indução por meio do dispositivo intravaginal CIDR-G ${ }^{\circledR 5}$.

Logo após a retirada dos dispositivos intravaginais, que ocorreu no quinto dia após a inserção, as fêmeas foram monitoradas a cada seis horas, no intuito de detectar o início do estro, com o auxílio de macho cirurgicamente preparado (rufião por translocação peniana). Os sinais de estro observados foram: procura pelo

\footnotetext{
${ }^{1}$ Advanced $^{\circledR}$ - Agroceres

${ }^{2}$ Progespon ${ }^{\circledR}$, Syntex S.A Indústria Bioquímica \&

Farmacêutica, Representante no Brasil Tecnopec.

${ }^{3}$ Estrogin ${ }$, Farmavet Ltda.

${ }^{4}$ Novormon ${ }^{\circledR}$, Tecnopec Ltda.

${ }^{5}$ Eazi-Breed CIDR-G ${ }^{\circledR}$, Pharmacia Saúde Animal do Brasil Ltda
} 
macho, inquietação, vocalização, micção freqüente, batimento de cauda, contração, hiperemia e edema de vulva, descarga de muco vaginal e imobilidade à monta, características estas consideradas como início do estro.

As fêmeas foram submetidas à monta natural 12 horas após detectado o estro e 24 horas depois, se continuassem em estro, utilizando-se dois machos de fertilidade comprovada, por meio de exame andrológico (CBRA, 1998) e histórico anterior de cobrições. Decorridos 35 dias depois da monta natural, todas as fêmeas foram avaliadas por ultra-sonografia trans-retal para detecção precoce da gestação.

Imediatamente após o início do estro, iniciaramse os exames ultra-sonográficos, a cada seis horas até 12 horas após a detecção da ovulação estro. As imagens dos ovários foram obtidas por via transretal (Menchaca e Rubianes, 2001) com o auxílio de um ultra-som, marca Aloka, modelo SSD-500, acoplado a um transdutor linear de $5 \mathrm{MHz}$. Os procedimentos para localização dos ovários foram os mesmos preconizados por Ginther e Kot (1994). O diâmetro, a posição e as características das estruturas dos ovários foram anotadas em fichas individuais. A emergência da onda folicular foi definida como sendo de um pool de pequenos folículos com diâmetro médio entre 3,0 e $4,0 \mathrm{~mm}$, que deram origem a um ou mais folículos com diâmetros maior ou igual a $5,0 \mathrm{~mm}$. O dia da emergência da onda foi aquele no qual o folículo apresentava $3,0 \mathrm{~mm}$ de diâmetro seguido por aumento em seu diâmetro para valores iguais ou superiores a $4,0 \mathrm{~mm}$ (Rubianes et al., 2003).

O diâmetro folicular foi obtido pela média da maior distância $(\mathrm{mm})$ entre dois pontos da cavidade antral dos folículos a partir de $2,5 \mathrm{~mm}$. A taxa de crescimento do folículo ovulatório ( $\mathrm{mm} /$ dia) foi obtida por meio da diferença dos diâmetros final e inicial, dividido pelo número de horas e multiplicado por 24 , a fim de alcançar a taxa de crescimento diário. A ovulação foi calculada pela média entre o horário da última visão do folículo ovulatório e o horário onde se detectou a ovulação.

As amostras de sangue foram coletadas por punção da veia jugular nos dias zero, um, três, cinco e seis, em tubos vacuolizados de $15 \mathrm{ml}$ com solução anticoagulante (EDTA) e acondicionados em caixa de isopor com banho de gelo. Os tubos foram imediatamente centrifugados a 3.000rpm durante 15 minutos, para a separação do plasma, que foi transferido para tubetes plásticos previamente identificados, e estocados à temperatura de $-20^{\circ} \mathrm{C}$, até sua análise. A concentração plasmática de progesterona foi determinada pela técnica de radioimunoensaio de fase sólida (RIA), utilizando-se kits comerciais $^{6}$, seguindo as recomendações do fabricante. A sensibilidade do teste era de $0,03 \mathrm{ng} / \mathrm{ml}$ e o coeficiente de variação intra-ensaio foi de $13,2 \%$ para $0,38 \pm 0,05 \mathrm{ng} / \mathrm{ml}$, $4,0 \%$ para $3,7 \pm 0,13$ e $5,3 \%$ para $17,0 \pm 0,90 \mathrm{ng} / \mathrm{ml}$. $\mathrm{O}$ coeficiente interensaio foi de $14,8 \%$ para $0,27 \pm 0,04 \mathrm{ng} / \mathrm{ml}, 4,3 \%$ para $3,5 \pm 0,15 \mathrm{ng} / \mathrm{ml}$ e $5,6 \%$ para $16,0 \pm 0,90 \mathrm{ng} / \mathrm{ml}$.

$\mathrm{Na}$ análise estatística utilizou-se o programa SAEG (Sistema..., 1997). A fim de verificar a normalidade e a homogeinidade das variáveis estudadas empregaram-se os testes Lilliefors e Cochran e Barttlet, respectivamente. Aplicou-se a análise de variância para verificar as diferenças entre as variáveis estudadas. Quando presentes, as diferenças foram testadas utilizando-se o teste de médias Student Newman Keuls. As variáveis que não apresentavam normalidade ou homogeinidade mesmo após a transformação logaritímica foram submetidas à análise nãoparamétrica e as médias foram comparadas pelo teste Wilcoxon (Sampaio, 2002).

\section{RESULTADOS}

No momento da inserção do dispositivo, somente uma cabra apresentou concentração de progesterona acima de $1 \mathrm{ng} / \mathrm{ml}$. Um dia após a retirada dos dispositivos, todas elas mostravam concentrações de progesterona inferiores a $1 \mathrm{ng} / \mathrm{ml}$.

Todas as cabras entraram em estro (Tab. 1), sendo a taxa de gestação de $83,3 \%(5 / 6)$ para $\mathrm{T} 1$ e de $50,0 \%(3 / 6)$ para T2. Cabras induzidas com esponja ou CIDR-G ${ }^{\circledR}$ entraram em estro $39,0 \pm 9,85$ e $35,0 \pm 5,89$ horas, após a retirada dos dispositivos, respectivamente $(\mathrm{P}>0,58)$.

\footnotetext{
${ }^{6}$ Coat-a-count $^{\circledR}$ progesterone Kit - DPC - Diagnostic Product Co., Los Angeles, CA, USA
} 
Tabela 1. Animais em estro (AE), taxa de gestação (TG), intervalos: da retirada dos dispositivos ao início do estro (IE) e da retirada dos dispositivos ao final do estro (IF) e duração do estro (DE) em cabras sincronizadas com esponjas intravaginais ou CIDR-G ${ }^{\circledR}($ média $\pm D P)$

\begin{tabular}{lccc}
\hline & T1 (esponja) & T2 $\left(\mathrm{CIDR}^{\mathrm{G}}{ }^{\mathbb{B}}\right)$ & Total (n) \\
\hline $\mathrm{AE}$ & $100 \%(6 / 6)$ & $100 \%(6 / 6)$ & $100 \%(12 / 12)$ \\
$\mathrm{TG}$ & $83,3 \%(5 / 6)$ & $50,0 \%(3 / 6)$ & $66,7,3 \%(8 / 12)$ \\
$\mathrm{IE}$ (horas) & $39,0 \pm 9,8 \mathrm{a}$ & $35,0 \pm 5,9 \mathrm{a}$ & $37,0 \pm 8,0$ \\
$\mathrm{IF}$ (horas) & $76,0 \pm 11,8 \mathrm{a}$ & $71,0 \pm 7,0 \mathrm{a}$ & $73,5 \pm 9,6$ \\
$\mathrm{DE}$ (horas) & $37,0 \pm 4,5 \mathrm{a}$ & $36,0 \pm 7,6 \mathrm{a}$ & $36,5 \pm 6,0$ \\
\hline
\end{tabular}

Médias com diferentes letras na mesma linha diferem entre si $(\mathrm{SNK} ; \mathrm{P}<0,05) . \mathrm{DP}=$ desvio-padrão.
A duração do estro não foi afetada pelo dispositivo utilizado $(\mathrm{P}>0,78)$, sendo $37,0 \pm 4,51$

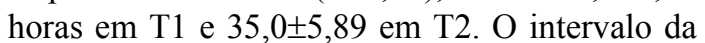
retirada do dispositivo ao final do estro não diferiu entre $\mathrm{T} 1$ e $\mathrm{T} 2$, sendo, respectivamente, $76,0 \pm 11,79$ e $71,0 \pm 7,02$ horas $(\mathrm{P}>0,60)$.

O número médio de ovulações $(1,50 \pm 0,84)$ foi o mesmo (Tab. 2), 1,50 $\pm 0,84$, para ambos os tratamentos $(\mathrm{P}>0,42)$.

Tabela 2. Número de ovulações por ciclo estral (NO), intervalos do início do estro à ovulação (IIO), do final do estro à ovulação (IFO) e da retirada do dispositivo à ovulação (IRO), diâmetro do folículo ovulatório (DFO) e taxa de crescimento do folículo ovulatório (TCFO) em cabras submetidas à indução do estro com esponjas intravaginais ou CIDR-G ${ }^{\circledR}$ (média $\left.\pm D P\right)$

\begin{tabular}{lccc}
\hline & T1 (esponja) & T2 $\left(\right.$ CIDR-G $\left.{ }^{\mathbb{B}}\right)$ & Média geral $(\mathrm{n})$ \\
\hline NO & $1,50 \pm 0,84 \mathrm{~A}$ & $1,5 \pm 0,84 \mathrm{~A}$ & $1,5 \pm 0,79$ \\
IIO (horas) & $29,0 \pm 5,9 \mathrm{a}$ & $25,0 \pm 7,0 \mathrm{a}$ & $27,06,5$ \\
IFO (horas) & $-8,0 \pm 3,1 \mathrm{a}$ & $-11,0 \pm 7,0 \mathrm{a}$ & $9,50 \pm 5,4$ \\
IRO (horas) & $68,0 \pm 12,4 \mathrm{a}$ & $60,0 \pm 10,7 \mathrm{a}$ & $64,0 \pm 11,8$ \\
DFO (mm) & $8,4 \pm 1,5 \mathrm{a}$ & $7,1 \pm 1,0 \mathrm{a}$ & $7,7 \pm 1,3$ \\
TCFO (mm/hora) & $2,9 \pm 1,7 \mathrm{a}$ & $1,7 \pm 1,3 \mathrm{a}$ & $2,2 \pm 1,6$ \\
\hline
\end{tabular}

Médias com diferentes letras minúsculas na mesma linha diferem entre si (SNK; $\mathrm{P}<0,05)$. Médias com diferentes letras maiúsculas na mesma linha diferem entre si (Wilcoxon; $\mathrm{P}<0,05)$. $\mathrm{DP}=$ desvio-padrão.

Os intervalos do início do estro à ovulação (IIO) foram $29,0 \pm 5,90$ e $25,0 \pm 7,01$ horas para $\mathrm{T} 1$ e $\mathrm{T} 2$, respectivamente $(\mathrm{P}>0,24)$. Assim como o $\mathrm{IIO}, \mathrm{o}$ intervalo final do estro à ovulação não foi influenciado pelo método de indução de estro empregado $(\mathrm{P}>0,21)$, isto é, $8,0 \pm 3,09$ e $11,0 \pm 7,01$ horas antes do término do estro em T1 e T2, respectivamente (Tab. 2).

O intervalo da retirada do dispositivo à ovulação não foi influenciado pelos tratamentos $(\mathrm{P}>0,22)$. Houve tendência de o diâmetro do folículo ovulatório ser maior em T1 $(\mathrm{P}<0,06)$. De forma semelhante, a menor taxa de crescimento observada em T2 $(0,17 \pm 0,13)$ tendeu a ser menor que T1 $(0,29 \pm 0,17)(\mathrm{P}<0,10)$.

\section{DISCUSSÃO}

A porcentagem total de animais em estro foi $100 \%$, o que mostra a eficiência dos dois procedimentos de indução. Diferentes estudos têm demonstrado a eficiência da associação entre progestágenos e gonadotrofinas na indução do estro em caprinos. Fonseca (2002), durante a estação de transição, obteve $90,6 \%$ de animais em estro, ao utilizar esponjas impregnadas com $60 \mathrm{mg}$ de MAP por nove dias. Prosperi et al. (2003), ao trabalharem durante a estação de anestro reprodutivo, encontraram taxa de indução média de 88,9\% utilizando esponjas impregnadas com a mesma quantidade de MAP por seis ou nove dias, enquanto Baril et al. (1993) obtiveram índices ligeiramente superiores (98\%), utilizando esponjas impregnadas com 50mg de acetato de fluorgesterona, porém com tempo de permanência de 11 dias. Assim como a esponja, o CIDR-G ${ }^{\circledR}$ induziu $100 \%$ de animais ao estro. Resultados semelhantes foram encontrados por Rubianes et al. (1998).

A taxa de gestação obtida após a indução com CIDR-G ${ }^{\circledR}, 50 \%$, foi inferior às descritas por Rubianes et al. (1995), 64\% $(n=14)$, e por Rubianes et al. (1998), 84\% $(\mathrm{n}=19)$. A taxa de gestação das cabras do $\mathrm{T} 1$ foi superior às observadas por Baril et al. (1993) e Fonseca (2002) $(n=118)$ e inferiores às relatadas por Prosperi et al. (2003) em cabras da raça Saanen 
$(n=16)$. Nesses experimentos, ocorreu $100 \%$ de taxa de gestação com o uso da esponja impregnada com 60mg de MAP por seis dias associado à aplicação de 250UI de eCG.

Carnevali et al. (1997) relataram somente 15\% de taxa de gestação em cabras da raça Cashmere. Para o autor, o uso de programas de indução de estro associado à monta natural deve estar condicionado a exames andrológicos dos machos. Assim como as fêmeas, os machos apresentam anestro estacional e sua utilização, nessas condições, pode levar à baixa taxa de gestação.

Ungerfeld e Rubianes (2002), ao compararem a utilização de CIDR-G $^{\circledR}$ e de esponjas impregnadas com MAP e fluogesterona em ovelhas, não observaram efeito sobre características reprodutivas no tempo de exposição de seis dias. Nesse mesmo estudo, quando se reduziu o tempo de exposição de 14 para seis dias, não houve modificação da taxa de gestação. Os dados deste estudo confirmaram os observados em ovelhas (Ungerfeld e Rubianes, 2002) e cabras (Prosperi et al., 2003), indicando que a redução do tempo de exposição à progesterona e aos progestágenos é uma alternativa viável aos tradicionais protocolos de indução do estro.

O intervalo da retirada do dispositivo ao início do estro foi de 37 horas, ou seja, similares ao obtido por Rubianes et al. (1998). Segundo Baril et al. (1996) e Rubianes et al. (1998), a fertilidade é reduzida quando esse intervalo é superior a 30 horas, contudo, isto não foi evidenciado nesta pesquisa. Esses autores relataram que a redução está possivelmente relacionada à presença de anticorpos anti-eCG. Também observaram maior intervalo do início do estro à retirada da esponja, e redução significativa na taxa de gestação em cabras que apresentaram porcentagem de ligação dos anticorpos ao eCG superior a 10. O maior intervalo observado apresenta causas diferentes das descritas por Baril et al. (1996), pois as cabras submetidas à indução não haviam recebido aplicação anterior de eCG. O efeito negativo de repetidas aplicações de eCG sobre os índices reprodutivos tem sido extensivamente relatado na literatura (Baril et al., 1993; Freitas et al., 1997). Uma alternativa ao uso do eCG em rebanhos em que se utilizaria intensivamente a indução do estro por via hormonal seria a utilização da gonadotrofina coriônica humana (hCG) em substituição ao eCG, conforme descrito por Machado e Simplício (2001).

O tipo de dispositivo utilizado não apresentou efeito sobre as características reprodutivas estudadas. Resultados semelhantes foram observados por Robin et al. (1994). O número de ovulações verificadas foi superior ao descrito por Fonseca (2002), que trabalhou com cabras da raça Alpina, e inferior em cabras das raças Alpinas e Saanen (Freitas et al. 1997).

O intervalo da retirada dos dispositivos à ovulação merece bastante atenção, pois a maioria dos protocolos de inseminação artificial por tempo fixo recomenda que a inseminação seja realizada entre 43 e 48 horas após a retirada do dispositivo (Leboeuf et al., 2000). Partindo do pressuposto de que a vida média dos espermatozóides que passaram pelo processo de congelamento/descongelamento é inferior, associado ao intervalo médio de 64 horas obtido, infere-se que as inseminações realizadas no intervalo recomendado possivelmente culminaram em baixa taxa de gestação. De fato, estudos realizados por Ritar et al. (1990) e Machado e Simplício (2001) mostraram que inseminações feitas precocemente ou tardiamente levaram à redução na taxa de fertilidade.

Poucos são os estudos sobre a dinâmica folicular e hormonal após a indução do estro em caprinos, e a maioria limita-se a relatar o intervalo até o início do estro e a taxa de gestação.

\section{CONCLUSÕES}

Ambos os protocolos estudados mostraram-se eficientes em induzir o estro. Desse modo, a escolha de um ou outro protocolo deve estar condicionada a outros fatores que não a eficiência reprodutiva.

\section{AGRADECIMENTOS}

À Dra. Marlene Bruschi, por ceder os animais e aos funcionários da Granja Água Limpa e da EMBRAPA-Gado de Leite. 


\section{REFERÊNCIAS BIBLIOGRÁFICAS}

BARIL, G.; LEBOEUF, B.; SAUMANDE, J. Synchronization of estrus in goats: the relationship between time of occurrence of estrus and fertility following artificial insemination. Theriogenology, v.40, p.621-628, 1993

BARIL, G.; REMY, B.; LEBOEUF, B. et al. Synchronization of estrus in goats: the relationship between eCG binding in plasma, time of occurrence of estrus and fertility following artificial insemination. Theriogenology, v.45, p.1553-1559, 1996.

CARNEVALI, F.; SCHINO, G.; DIVERIO, S. et al. Oestrus induction and synchronization during anoestrus in cashmere goats using hormonal treatment in association with "male effect". Europ. Fine Fibre Net., v.6, p.55-63, 1997.

CORDEIRO, P.R.C. Sincronização de cio em cabras leiteiras com fotoperiodismo artificial. In: CONGRESSO BRASILEIRO DE MEDICINA VETERINARIA, 22., 1992, Curitiba. Anais... Curitiba: Sociedade Goiana de Veterinária, 1992. p.22-25.

DEVESON, S.L.; FORSYTH, I.A.; ARENDT, J. Induced out-of-season breeding in British Saanen dairy goats: use of artificial photoperiods and/or melatonin administration. Anim. Reprod. Sci., v.29, p.1-15, 1992 .

FONSECA, J.F. Controle e perfil hormonal do ciclo estral e performance reprodutiva de cabras alpina $e$ saanen. 2002. 107f. Tese (Doutorado em Zootecnia) Departamento de Zootecnia, Universidade Federal de Viçosa, Viçosa.

FOOTE, W.C. Hormonal control of reproduction in sheep and goats. Dairy Goat J., v.60, p.560-568, 1982.

FREITAS, V.J.F.; BARIL， G.; SAUMANDE，J. Estrus synchronization in dairy goats: use of fluorogestone acetate vaginal sponges or norgestomet ear implants. Anim. Reprod. Sci., v.46, p.237-244, 1997.

GINTHER, O.J.; KOT, K. Follicular dynamics during the ovulatory season in goats. Theriogenology, v.42, p.987-1001, 1994.

HAWK, H.W.; CONLEY, H.H. Investigation of sperm transport failures in ewes administered synthetic progestagen. J. Anim. Sci., v.34, p.609-613, 1972.

LEBOEUF, B.; RESTALL, B.; SALAMON, S. Production and storage of goat semen for artificial insemination. Anim. Reprod. Sci., v.62, p.113-141, 2000.

MACHADO, R.; SIMPLICIO, A.A. Avaliação de programas hormonais para a indução e sincronização do estro em caprinos. Pesq. Agrop. Bras., v.36, p.171$178,2001$.
MANUAL para exame andrológico e avaliação de sêmen animal. 2.ed. Belo Horizonte: CBRA, 1998. $49 \mathrm{p}$.

MENCHACA, A.; RUBIANES, E. Effect of high progesterone concentrations during the early luteal phase on the lenght of the ovulatory cycle of goats. Anim. Reprod. Sci., v.68, p.69-76, 2001.

MIHM, M.; BAGUISI, A.; BOLAND, M.P. et al. Association between the duration of dominance of the ovulatory follicle and pregnancy rate in beef heifers. $J$. Reprod. Fertil., v.102, p.123-130, 1994.

OLIVIERA, M.A.L.; GUIDO, S.I.; LIMA, P.F. Comparison of different protocols used to induce and synchronize estrus cycle of Saanen goats. Small Rum. Res., v.40, p.149-153, 2001.

PROSPERI, C.P.; TORRES, C.A.A.; MAFFILI, V.V. et al. Indução do estro em cabras Saanen nulíparas, utilizando-se diferentes tempos de exposição ao progestágeno. Rev. Bras. Reprod., v.27, p.481-483, 2003.

RITAR, A.J.; BALL, P.D.; O'MAY, P.J. Artificial insemination of Cashmere goats: effects on fertility and fecundity of intravaginal treatment, method and time of insemination, semen freezing process, number of motile spermatozoa and age of females. Reprod. Fertil. Devel., v.2, p.377-384, 1990.

ROBIN, N.; LAFOREST, J.P.; LUSSIER, J.G. et al. Induction of estrus with intramuscular injections of GnRH or PMSG in lactating goats (Capra hircus) primed with a progestagen during seasonal anestrus. Theriogenology, v.42, p.107-116, 1994.

RUBIANES, E.; DE CASTRO, T.; KMAID, S. Estrous response after a short progesterone priming in seasonally anestrous goats. Theriogenology, v.49, p.345, 1998. Abstract.

RUBIANES, E.; MENCHACA, A. The pattern and manipulation of ovarian follicular growth in goats. Anim. Reprod. Sci., v.78, p.271-287, 2003.

RUBIANES, E.; MENCHACA, A.; CARBAJAL, B. Response of the 1-5 day-aged ovine corpus luteum to prostaglandin F2 $\alpha$. Anim. Reprod. Sci., v.78, p.47-55, 2003.

SAMPAIO, I.B.M. Estatística aplicada à experimentação animal. Belo Horizonte:FEPMVZEditora, 2002. 265p.

SISTEMA de análises estatísticas e genéticas - SAEG. Versão 8.0.Viçosa, MG:UFV, 1997. 150p.

UNGERFELD, R.; RUBIANES, E. Short term priming with different progestogen intravaginal devices (MAP, FGA, CIDR-G) for eCG-estrous induction in anestrus ewes. Small Rum. Res., v.46, p.63-66, 2002. 